\title{
Local orbitals approach to the anomalous Hall and Nernst effects in itinerant ferromagnets
}

\author{
Pavel Středa ${ }^{\mathrm{ab}}$ \\ Institute of Physics ASCR, Na Slovance 2, 18221 Praha 8, Czech Republic
}

\begin{abstract}
Linear response of the orbital momentum to the gradient of the chemical potential is used to obtain anomalous Hall conductivity. Transition from the ideal Bloch system for which the conductivity is determined by the Berry phase curvatures to the case of strong disorder for which the conductivity becomes dependent on the relaxation time is analysed. Presented tight-binding model reproduces experimentally observed qualitative features of the anomalous Hall conductivity and the transverse Peltier coefficient in the so called bad-metal and scattering-independent regimes.
\end{abstract}

\section{Introduction}

Ferromagnetic materials exhibit, in addition to the standard Hall, an extraordinary Hall effect which does not vanish at zero magnetic field. The quantum theory of this so-called anomalous Hall effect has been traditionally based on the Kubo-formula, i.e. on the response to the dynamical force, the electric field [1]. General theory of irreversible processes suggests that within the linear approach the same result has to be obtained by the response of the system to the statistical force, gradient of the chemical potential $\mu$. By using this response the relation between asymmetric part of the conductivity tensor and the orbital momentum can easily be derived, as shown in Section 2 . In the pure crystal limit the obtained expression for the anomalous Hall conductivity is equivalent to the Kubo-formula result which expresses the conductivity in terms of the Berry phase curvatures $[1,3]$.

Three broad regimes of the anomalous Hall conductivity have been identified when surveying a large body of experimental data for diverse materials: so called bad-metal, scattering-independent and high conductivity regimes [1, 2]. In the first two regimes the side-jump scattering [4] dominates. In the high conductivity regime the transverse current is proportional to the longitudinal current. This property is commonly attributed to the asymmetric part of scattering, the so called skew scattering [5]. We limit our attention to the systems for which the statistically averaged Green functions are invariant under the crystal translations. Since skew scattering violates this condition the high conductivity regime is excluded from the treatment. Presented analysis is thus limited to the bad-metal and scatteringindependent regimes.

To analyse scaling of the anomalous Hall conductivity and the transverse Peltier coefficient with the longitudinal conductivity a simple tight-binding model is used. Two independent fully spin-polarized bands are considered. Each of them is supposed to be formed by atomic-like states hav-

e-mail: streda@fzu.cz

a The author acknowledge support from the Czech Science Fqundation, Project P204/11/1228. ing the same orientation of orbital momentum but opposite for different bands. Finite relaxation time is supposed to originate in fluctuation of atomic state energies. The detail model description is presented in Section 3. The following Section 4 is devoted to the obtained scaling of the anomalous Hall conductivity and Peltier coefficient. Main results together with the possible applicability to real systems are summarised in the last Section.

\section{Response of the orbital momentum to the gradient of the chemical potential}

The non-zero magnetization of ferromagnetic materials is attributed to the existence of spin-polarized bands. Within the tight-binding approach these bands originate in a network of overlapping atomic, or more generally molecular, spin-polarized orbitals having non-zero orbital momentum as sketched in Fig. 1. The resulting orbital momentum density of electrons, represented by the Hamiltonian $H$, is defined as follows

$$
\mathbf{L}=m_{0} \operatorname{Tr}\left[f_{0}(H-\mu) \mathbf{r} \times \mathbf{v}\right] \equiv \int \mathbf{L}(\mathbf{r}) d^{3} r,
$$

where $f_{0}(H-\mu)$ denotes the Fermi-Dirac distribution operator. It gives rise to the circulating current density

$$
\mathbf{j}(\mathbf{r})=\frac{e}{m_{0}} \operatorname{curl} \mathbf{L}(\mathbf{r}),
$$

where $e$ and $m_{0}$ are the absolute value of the electron charge and mass, respectively. For finite samples surface currents due to modification of orbitals by the surface vicinity can also appear. However, this phenomenon is limited to a surface layer and the resulting contribution thus scales with surface area. It vanishes in the thermodynamic limit of the infinite sample volume. This is the essential difference from the case of orbits induced by the external magnetic field giving rise surface diamagnetic currents. Their contribution scales with the sample volume as discussed in detail in connection with the quantum Hall effect [6]. 


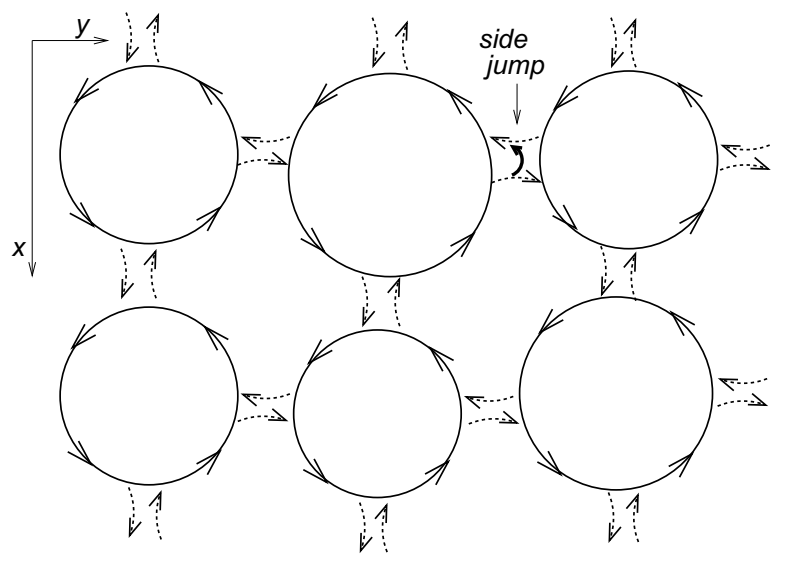

Fig. 1. Scheme of the atomic (molecular) orbital network within an atomic plane perpendicular to the sample magnetization. Hopping vanishes at the left hand side of the network representing the sample surface.

Within the linear response localized current loops cannot give rise to any net current by their definition. Only the part of orbital momentum, $\mathbf{L}_{\mathrm{fc}}$, originating in the so called free currents is responsible for a Hall current induced by the chemical potential gradient $\nabla \mu$

$$
\mathbf{j}_{H}=-\frac{e^{2}}{m_{0}} \frac{d \mathbf{L}_{\mathrm{fc}}}{d \mu} \times \frac{\nabla \mu}{e} .
$$

It defines the anti-symmetric part of the conductivity tensor [7]. Choosing $z$-axis to be parallel with $\mathbf{L}_{\mathrm{fc}}$ the Hall conductivity in the zero temperature limit reads

$$
\sigma_{x y}(\mu)=e^{2} \operatorname{Tr}\left[\delta(H-\mu)(\mathbf{r} \times \mathbf{v})_{z}\right]_{\mathrm{fc}},
$$

where the lower index (fc) limits the trace to the subspace of extended Fermi-electron states representing free currents.

For perfect Bloch systems all states represented by a wave vector $\mathbf{k}$ are extended and the Hall conductivity given by Eq. (4) can be written as

$$
\sigma_{x y}^{(\mathrm{pBs})}(\mu)=\frac{e^{2}}{8 \pi^{3}} \sum_{n} \int \frac{d f_{0}}{d \mu}\left[\mathbf{r}_{n}(\mathbf{k}) \times \mathbf{v}_{n}(\mathbf{k})\right]_{z} d^{3} k,
$$

where $n$ stands for the band index and $\mathbf{v}_{n}(\mathbf{k})$ is the expectation value of the velocity operator, $\mathbf{v}_{n}(\mathbf{k})=\nabla_{\mathbf{k}} E_{n}(\mathbf{k}) / \hbar$. Because of the system periodicity the expectation value of the coordinate $\mathbf{r}$ can be written as

$$
\mathbf{r}_{n}(\mathbf{k})=\frac{1}{\Omega} \int_{\Omega} u_{n, \mathbf{k}}^{+}(\mathbf{r}) \mathbf{r} u_{n, \mathbf{k}}(\mathbf{r}) d^{3} r+\frac{1}{N} \sum_{l=1}^{N} \mathbf{R}_{l},
$$

where $u_{n, \mathbf{k}}(\mathbf{r})$ stands for the periodic part of the Bloch function, $\Omega$ denotes the unit cell volume and $N=1 / \Omega$ is the number of unit cells per unit volume. While $\mathbf{r}_{n}(\mathbf{k})$ depends on the choice of the coordinate origin the sum of vector products $\mathbf{r}_{n}(\mathbf{k}) \times \mathbf{v}_{n}(\mathbf{k})$ does not since the total velocity vanishes in equilibrium. Approximate expectation value of $\mathbf{r}$ is given by the radius vector of the local orbitals.

The total orbital momentum of electrons can be divided into two parts. The contribution given by electron motion around local orbitals is determined by vector products of inter-band matrix elements, $\mathbf{r}_{n, n^{\prime}}(\mathbf{k}) \times \mathbf{v}_{n^{\prime}, n}(\mathbf{k})$. It does not contribute to the Hall conductivity. The remaining free current contribution originates in the separation of masscentre positions of $\mathbf{k}$ - and -k-states. It is controlled by the real-space distribution of their electron densities [8]. Their different occupation leads to a local charge polarization [9, 10]. The corresponding Fermi electron contribution to the orbital momentum can thus be understood as the orbital polarization momentum. It is equivalent to the Fermi electron contribution of the Berry-phase correction to the orbital magnetization [11]. It is only this contribution that determines the Hall conductivity.

Note that the most effective scattering leading to electron transitions from a $\mathbf{k}$-state to the - $\mathbf{k}$-state is of the sidejump character because of the space separation of their mass-centre positions, as sketched in Fig. 1.

The expression for the Hall conductivity of the perfect Bloch system, Eq. (5), is identical to that obtained by the use of the Kubo-formula [1], originally derived by Karplus and Luttinger [12]. Using the alternative form for the coordinate expectation value derived with the help of Wannier functions [13]

$$
\mathbf{r}_{n}(\mathbf{k})=-\operatorname{Im} \int u_{n, \mathbf{k}}^{+}(\mathbf{r}) \nabla_{\mathbf{k}} u_{n, \mathbf{k}}(\mathbf{r}) d^{3} r+\frac{1}{N} \sum_{l=1}^{N} \mathbf{R}_{l},
$$

the integration by parts over the Brillouin zone yields

$$
\sigma_{x y}^{(\mathrm{pBs})}(\mu)=-\frac{e^{2}}{h} \sum_{n} \frac{1}{4 \pi^{2}} \int_{\mathrm{BZ}} f_{0}\left(E_{n}(\mathbf{k})-\mu\right)\left[\mathbf{\Omega}_{n}(\mathbf{k})\right]_{z} d^{3} k,
$$

where $\boldsymbol{\Omega}_{n}(\mathbf{k})$ is just the Berry phase curvature [3]

$$
\boldsymbol{\Omega}_{n}(\mathbf{k})=-\operatorname{Im} \int\left[\nabla_{\mathbf{k}} u_{n, \mathbf{k}}^{+}(\mathbf{r})\right] \times\left[\nabla_{\mathbf{k}} u_{n, \mathbf{k}}(\mathbf{r})\right] d^{3} r
$$

\section{Tight-binding two-band model}

The aim of this section is to present a simple model system allowing to understand the main features of the anomalous Hall conductivity at zero external magnetic field. For the sake of simplicity the consideration will be limited to isotropic systems, like those of the cubic symmetry. Two spin-polarized bands defined by the following tight-binding model Hamiltonian will be considered

$$
H^{( \pm)}=\sum_{l}|l, \pm\rangle E_{l}^{( \pm)}\left\langle l, \pm\left|+\sum_{l, m}^{l \neq m}\right| l, \pm\right\rangle t_{l m}\langle m, \pm|,
$$

where $|l, \pm\rangle$ are Wannier functions representing atomic or molecular orbitals of the energy $E_{l}^{( \pm)}$associated with lattice sites $\mathbf{R}_{l}$. Spin-polarized orbitals are supposed to have nonzero $z$-component of the orbital momentum only, and its orientation is represented by the index $( \pm)$. The system disorder is modelled by considering a variation of orbital energies $E_{l}^{( \pm)}$around their mean values $E_{a}^{( \pm)}=\left\langle E_{l}^{( \pm)}\right\rangle$. The energy difference $E_{a}^{(+)}-E_{a}^{(-)}$represents an average Zeeman spin spliting. Hopping integrals $t_{l m}$ are supposed to be fluctuation-independent quantities. This so called diagonal disorder model covers a variety of possible fluctuations representing for example the alloy composition or a local variation of the Zeeman spliting. 
To establish longitudinal conductivity the Kubo-Greenwood formula is used [14]. Since for the considered diagonal disorder vortex corrections vanish it is given as follows

$$
\sigma_{x x}^{( \pm)}(\mu)=\frac{\pi e^{2} \hbar}{\tilde{a}^{3}} \int\left\langle\delta\left(\eta+E_{l}^{( \pm)}-\mu\right)\right\rangle_{\mathrm{av}}^{2} \Phi_{x x}(\eta) d \eta,
$$

where $\tilde{a}^{3}$ denotes the Wiegner-Seitz cell volume, angular brackets $\langle\cdots\rangle_{\mathrm{av}}$ represent statistical averaging and $\Phi_{x x}(\eta)$ is a disorder independent function given by energy dispersion $E(\mathbf{k})$ of the disorder-free system

$$
\Phi_{x x}(\eta)=\sum_{\mathbf{k}} v_{x}^{2}(\mathbf{k}) \delta(E(\mathbf{k})-\eta)
$$

Hall conductivity, given by Eq. (4), is determined by the orbital momentum where $\mathbf{r}$ within each of WiegnerSeitz cells is given as the sum of the position vector $\mathbf{R}_{l}$ of the orbital centre and $\mathbf{r}_{l}$ representing corresponding relative coordinate. Within the tight-binding model the expectation value $\mathbf{r}_{l}(\mathbf{k})$ can be approximated by the radius vector of orbitals. Since the orbital radius increases with increasing orbital energy we can write

$$
\mathbf{r}_{l}(\mathbf{k})= \pm r\left(E_{l}\right) \mathbf{e}(\mathbf{k}),
$$

where $\mathbf{e}(\mathbf{k})$ is the expectation value of the radius vector given by the orbit re-scaled to close the area just equal to $\pi$ and having the positive value of the orbital momentum.

Since in the equilibrium the total velocity vanishes the sum of $\mathbf{R}_{l} \times \mathbf{v}$ over allowed velocities vanishes as well. By using approximations described above the Hall conductivity, Eq. (4), can be written as follows

$$
\sigma_{x y}^{( \pm)}(\mu)= \pm \frac{e^{2}}{\tilde{a}^{3}} \int\left\langle\delta\left(\eta+E_{l}^{( \pm)}-\mu\right)\right\rangle_{\mathrm{av}} r(\eta) \Phi_{x y}(\eta) d \eta
$$

where

$$
\Phi_{x y}(\eta)=\sum_{\mathbf{k}}[\mathbf{e}(\mathbf{k}) \times \mathbf{v}(\mathbf{k})]_{z} \delta(E(\mathbf{k})-\eta) .
$$

The anomalous Nernst effect can be characterised by the transverse Peltier coefficient $\alpha_{x y}(\mu)$. In the low temperature limit the Sommerfeld expansion leads to the Mott rule $[15,16]$

$$
\alpha_{x y}^{( \pm)}(\mu)=\frac{\pi^{2}}{3} \frac{k_{B}^{2} T}{e}\left[\frac{d \sigma_{x y}^{( \pm)}(E)}{d E}\right]_{E=\mu},
$$

where $T$ and $k_{B}$ are the temperature and the Boltzmann constant, respectively.

To estimate transport coefficients, we have to make an additional assumption about the functions $\Phi_{\alpha \beta}(\eta)$. Let us assume that states within the lower half of a band are of the free-electron type with a constant effective mass while those in upper half of the band are of the hole-type with the same absolute value of the mass. The corresponding density of states normalised per Brillouin zone is given as follows

$$
\begin{array}{ll}
g(\eta)=\frac{c^{3 / 2}}{\sqrt{2} \pi^{2} w} \sqrt{1-|\eta| / w}, & |\eta| \leq w, \\
g(\eta)=0, & |\eta|>w,
\end{array}
$$

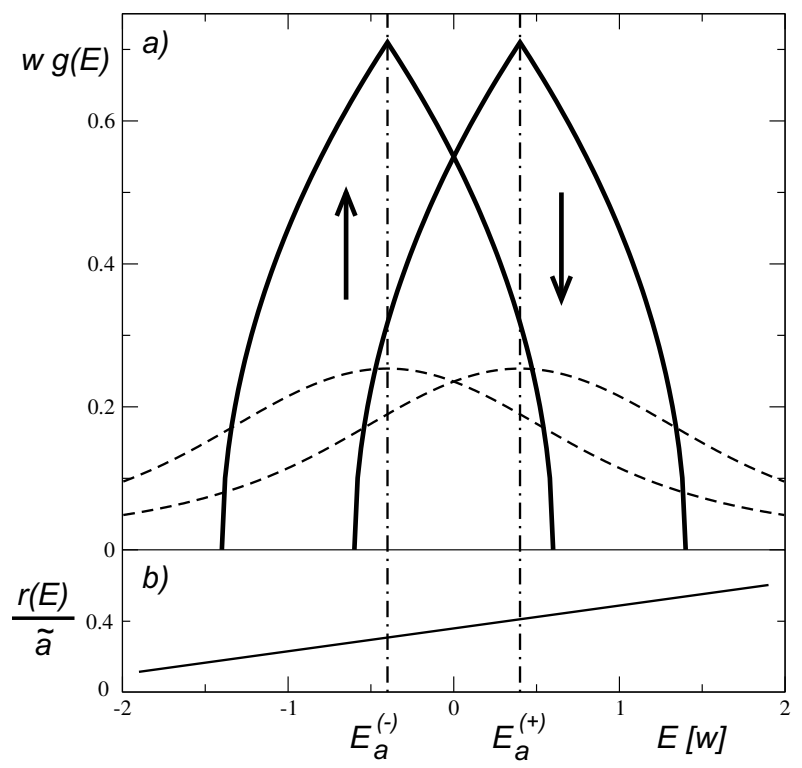

Fig. 2. a) Model density of states for two spin-polarized bands with $\Gamma=0$ (full lines) and $\Gamma / w=1$ (dashed lines). b) Energy dependence of the orbital radius.

where the normalisation constant $c=\left[3 \pi^{2} /(2 \sqrt{2}]^{2 / 3}\right.$ and $w$ is the half band width. The resulting energy dependence for two considered bands is shown in Fig. 2. Resulting estimates for $\Phi_{\alpha \beta}(\eta)$ read

$$
\frac{1}{\tilde{a}^{2}} \Phi_{x x}(\eta)=\frac{1}{3} \frac{4 \pi^{4}}{c^{4}} \frac{1}{\hbar^{2}} w^{4} g^{3}(\eta)
$$

and considering a circular shape of orbits

$$
\frac{1}{\tilde{a}} \Phi_{x y}(\eta)=\frac{1}{\hbar} \frac{\pi^{3}}{2 c^{2}} w^{2} g^{2}(\eta)
$$

Let us assume that the variation of orbital energies is controlled by a probability distribution $p\left(E_{l}^{( \pm)}-E_{a}^{( \pm)}\right)$. The simplest approach allowing to estimate the effect of this diagonal disorder is the so called virtual crystal approach which gives

$$
\left\langle\delta\left(\eta+E_{l}^{( \pm)}-\mu\right)\right\rangle_{\mathrm{av}}=p\left(\eta+E_{a}^{( \pm)}-\mu\right) .
$$

It leads to a band broadening as shown in Fig. 2 for the Gaussian distribution

$$
p_{G}(\eta, \Gamma)=\frac{1}{\Gamma \sqrt{2 \pi}} e^{-\frac{\eta^{2}}{2 \Gamma^{2}}}
$$

Within this approach the conductivity components read

$$
\sigma_{x x}^{( \pm)}(\mu)=\frac{e^{2}}{h \tilde{a}} \frac{8 \pi^{6} w^{4}}{3 c^{4}} \int_{-w}^{+w} p^{2}\left(\eta+E_{a}^{( \pm)}-\mu\right) g^{3}(\eta) d \eta,
$$

and

$$
\sigma_{x y}^{( \pm)}(\mu)= \pm \frac{e^{2}}{h \tilde{a}} \frac{\pi^{4} w^{2}}{c^{2} \tilde{a}} \int_{-w}^{+w} p\left(\eta+E_{a}^{( \pm)}-\mu\right) r(\eta) g^{2}(\eta) d \eta,
$$

where $r(\eta)$ is defined by Eq. (13). 


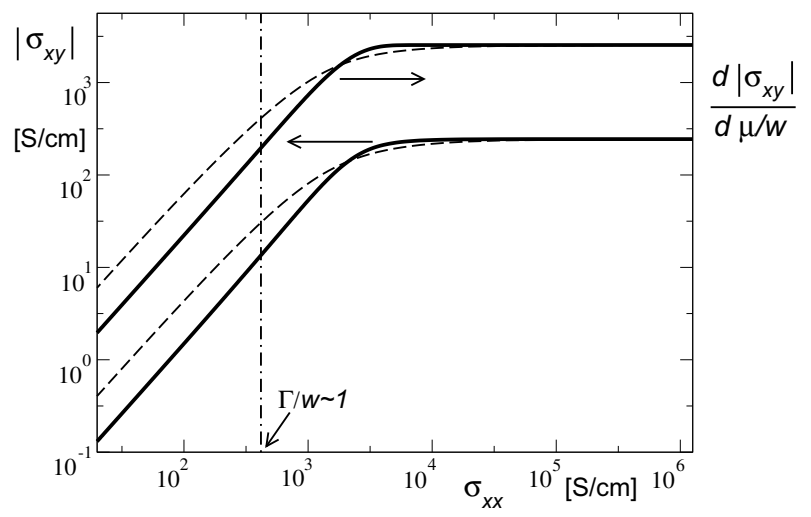

Fig. 3. Anomalous Hall conductivity $\sigma_{x y}$ (lower curves) and its derivative with respect of $\mu$ (upper curves) as function of the longitudinal conductivity $\sigma_{x x}$. Full lines correspond to the disorder represented by the Gaussian distribution of orbital energies fluctuations while dashed lines are for the Lorentz distribution.

\section{Results and discussion}

Typical dependence curves of the anomalous Hall conductivity and the Peltier coefficient, Eq. (16), on the longitudinal conductivity established by the use of the Eqs. (22) and (23) are presented in Fig. 3 for two considered distributions $p(\eta)$, Gaussian and Lorentz. Both bands are assumed to be of the same width, $2 w$, and the chemical potential is located just in the middle between mean orbital energies, $\mu=\left[E_{a}^{(+)}+E_{a}^{(-)}\right] / 2$. Such a model system corresponds to the so called compensated metal having the same concentration of electrons and holes at the Fermi level. In this case the longitudinal Peltier coefficient $\alpha_{x x}$ vanishes. Energy dependence of the orbit radius has been approximated by the linear expansion $r(\eta) / \tilde{a}=0.4(1+0.4 \eta / w)$ with $\tilde{a}=3 \AA$. For separation of mean orbital energies the value $0.8 w$ has been considered. The same parameters have been used for dependence presented in Fig. 2.

Two regimes can be identified in Fig. 3. First, it is the scattering-independent regime in which the anomalous Hall conductivity approaches a constant value for $\sigma_{x x}>$ $10^{4} \mathrm{~S} / \mathrm{cm}$. In this regime the band broadening $\Gamma$ is much smaller then unperturbed band width $w$ and $\sigma_{x y}$ for the perfect Bloch system is not significantly affected. Second, it is the bad-metal regime $\left(\sigma_{x x} \leq 10^{3} \mathrm{~S} / \mathrm{cm}\right)$ in which the anomalous Hall conductivity decreases with decreasing $\sigma_{x x}$ at a rate faster than linear. In this case of strong fluctuations, $\Gamma / w \geq 1$, the band broadening becomes substantial. The resulting overlap of spin-polarized bands leads to the suppression of the total orbital momentum and consequently the Hall conductivity. The numerical example with the used Gaussian distribution gives $\sigma_{x y} \sim \sigma_{x x}^{1.57}$ in the region for which $\Gamma / w \sim 1$.

The established scaling is in qualitative agreement with that observed experimentally for several quite different ferromagnetic materials [2]. For example, $\mathrm{Cu}_{1-x} \mathrm{Zn}_{x} \mathrm{Cr}_{2} \mathrm{Se}_{4}$ in the bad-metal regime gives $\sigma_{x y} \sim \sigma_{x x}^{1.6}$ which is close to the presented model result. Such agreement seems to be surprising because of the model simplicity. Especially the virtual crystal approach representing the effect of the potential fluctuations is a crude approximation. Nevertheless it has been found that more sophisticated approaches do not change the qualitative behaviour presented. It indicates the universality of the scaling.

\section{Concluding remarks}

It has been shown that anomalous Hall conductivity is determined by the part of orbital momentum given by free electron currents, orbital polarization momentum. For a perfect Bloch system it is equivalent to the Fermi electron contribution of the Berry-phase corrections to the orbital magnetization [11]. General properties of the anomalous Hall conductivity and the transverse Peltier coefficient have been presented by using the simple two-band model based on the local orbital approach. Despite of its simplicity it qualitatively describes the scaling of the anomalous Hall conductivity with the longitudinal conductivity within bad-metal and scattering-independent regimes.

To estimate anomalous Hall conductivity for a real material requires knowledge of local orbitals represented by Wannier functions and also the specific form of fluctuations relevant for the studied system. It is a challenge to work out such a procedure based on the first principle calculations. Newly developed numerical techniques allowing to establish Wannier functions giving the best tight-binding model parameters $[17,18]$ or the linear muffin-tin orbital (LMTO) method [19] together with the coherent potential approximation [20] seem to be a proper way to establish anomalous Hall effect for real materials.

\section{References}

1. N. Nagaosa, J. Sinova, S. Onoda, A. H. MacDonald and N. P. Ong, Rev. Mod. Phys. 82, (2010) 1539

2. T. Miyasato, N. Abe, T. Fujii, A. Asamitsu, S. Onoda, Y. Onose, N. Nagaosa, and Y. Tokura, Phys. Rev. Lett. 99, (2007) 086602

3. G. Sundaram and Q. Niu, Phys. Rev. B 59, (1999) 14915

4. L. Berger, Phys. Rev. B 2, (1970) 4559

5. J. Smit, Physica 21, (1955) 877; 24, (1958) 39

6. P. Středa, J. Phys. C: Solid State Phys. 16, (1983) L895

7. L. D. Landau and E. M. Lifshitz, Electrodynamics of continuous media (Pergamon, Oxford, 1960) p.97, eq.(21.5)

8. P. Středa, and T. Jonckheere, Phys. Rev. B 82, (2010) 113303

9. E. N. Adams, and E. I. Blount, J. Phys. Chem. Solids 10, (1959) 286

10. R. C. Fivaz, Phys. Rev. 183, (1969) 586

11. D. Xiao, Y. Yao, Z. Fang, and Q. Niu, Phys. Rev. Lett. 97, (2006) 026603

12. R. Karplus, and J. M. Luttinger, Phys. Rev. 95, (1954) 1154

13. P. Středa, Phys. Rev. B 82, (2010) 045115

14. D. A. Greenwood, Proc. Phys. Soc. (London) 71, (1958) 585

15. E. H. Sondheimer, Proc. R. Soc. London 193, (1948) 484

16. L. Smrčka and P. Středa, J. Phys. C 10, (1977) 2153

17. N. Marzari, and D. Vanderbilt, Phys. Rev. B 56, (1997) 12847

18. X. Wang, J. R. Yates, I. Souza, and D. Vanderbilt, Phys. Rev. B 74, (2006) 195118

19. I. Turek, J. Kudrnovský, V. Drchal, L. Szunyogh, and

P. Weinberger, Phys. Rev. B 65, (2002) 125101

20. B. Velick y, Phys. Rev. 184, (1969) 614 